\title{
High in-hospital cardiac arrest rate in a district general
}

hospital

Eid Hussien, SAS Anaesthetics, South Tyneside NHS Foundation Trust. Christian Frey, Consultant Anaesthetist, South Tyneside NHS Foundation Trust.

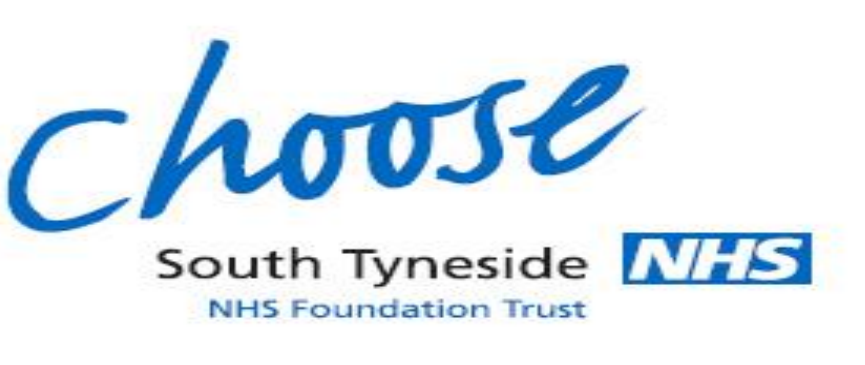

\section{Why this audit?}

- The National Cardiac Arrest Audit (NCAA) for the period $1 / 4 / 2015-31 / 3 / 2016$ revealed a higher than expected number of cardiac arrests per 1000 hospital admissions at our Trust compared to national average (1).

- 44 cardiac arrests on hospital wards against a national average number of 30 .

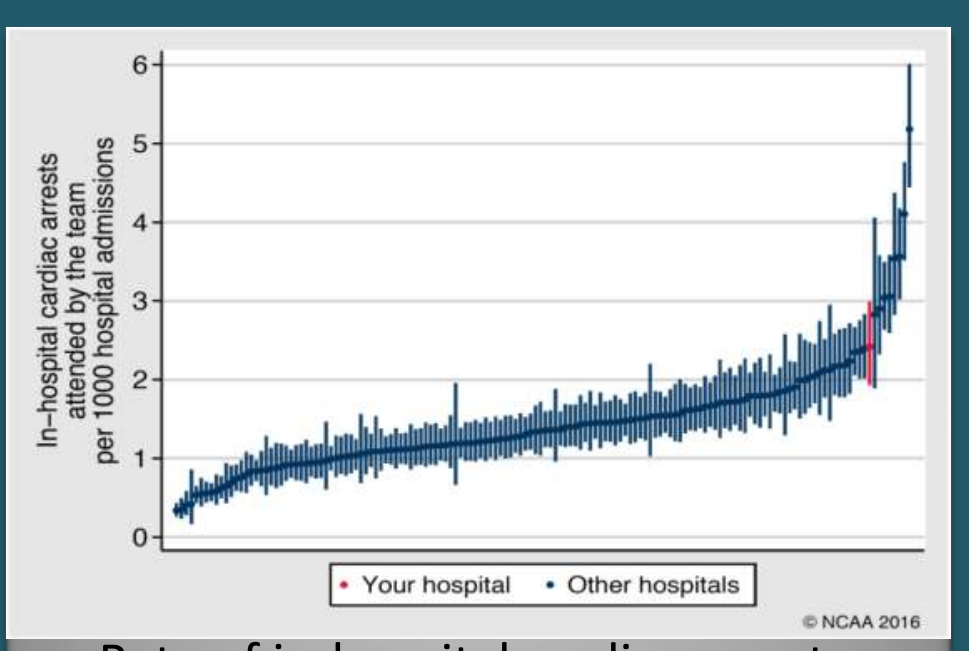

Rate of in-hospital cardiac arrests

\section{Aims and Objectives}

- To determine standards of care up to the cardiac arrest and potential for preventability.

\section{Specific criteria/standards}

- Pre-arrest observations intervals were traced and compared to hospital policy.

- Time intervals between ITU outreach/ medical team request and review were observed.

- Overall clinical management was measured against NCEPOD and Hogan scoring systems. $(2,3)$

\section{Methodology}

Multidisciplinary retrospective analysis of retrieved patients' notes. All notes reviewed separately and independently by ITU/Anaesthetics specialty doctor level or above and critical care outreach nurse. Case notes could be obtained for 38 of the 44 cardiac arrests.

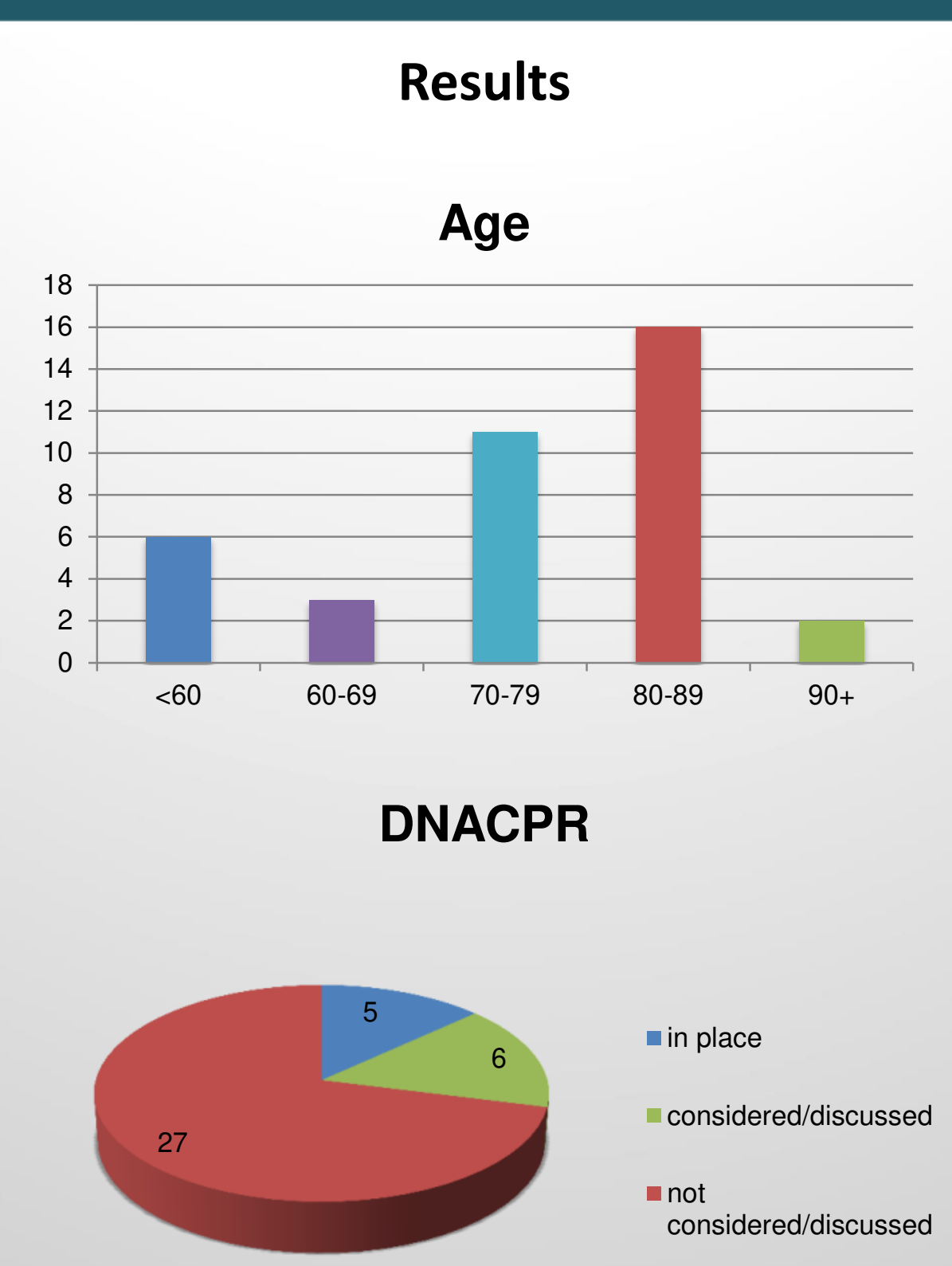

\section{Observations before arrest}

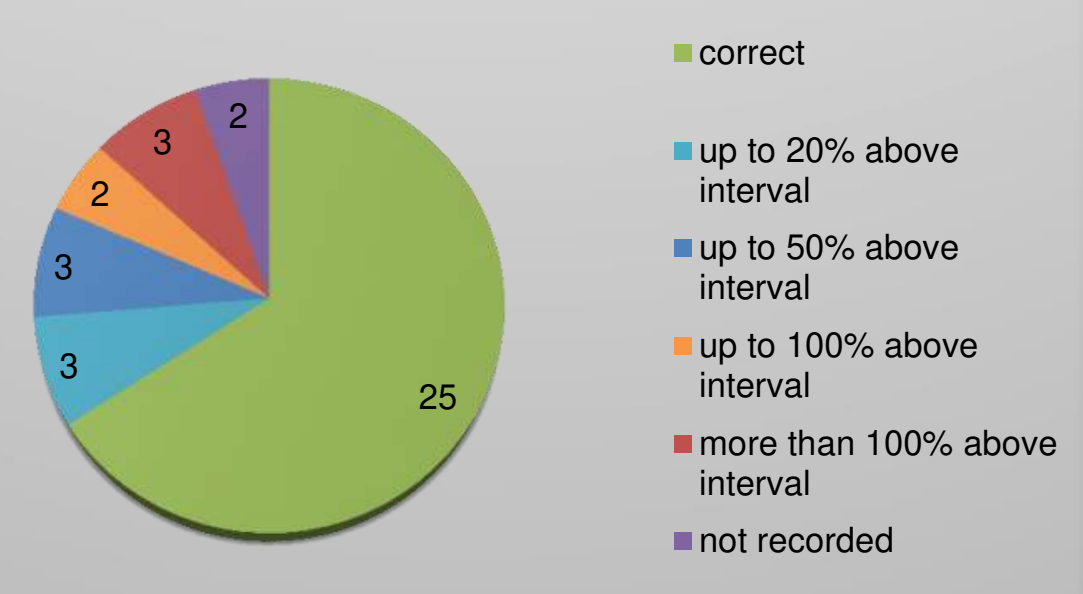

Observations, NCEPOD, HOGAN Scores and outcome

- Most of the observations were done within the right time frame.

- Hogan score showed $>2 / 3$ of cases with no evidence or slight evidence of preventability for both nurses and doctors.

- NCEPOD scoring for both doctors and nurses showed $<5 \%$ of cases were good clinical practice; $8 \%$ (doctors assessment) and $17 \%$ (nurses assessment) were deemed "less than satisfactory".

- 16 cases out of 38 developed ROSC.

- 12 cases out of 16 who had ROSC deemed inappropriate/ didn't require ITU admission.

- 3 cases out 16 who had ROSC were discharged home alive.

- $>60 \%$ (23) of cardiac arrest cases were admitted into the hospital for $>3$ days.

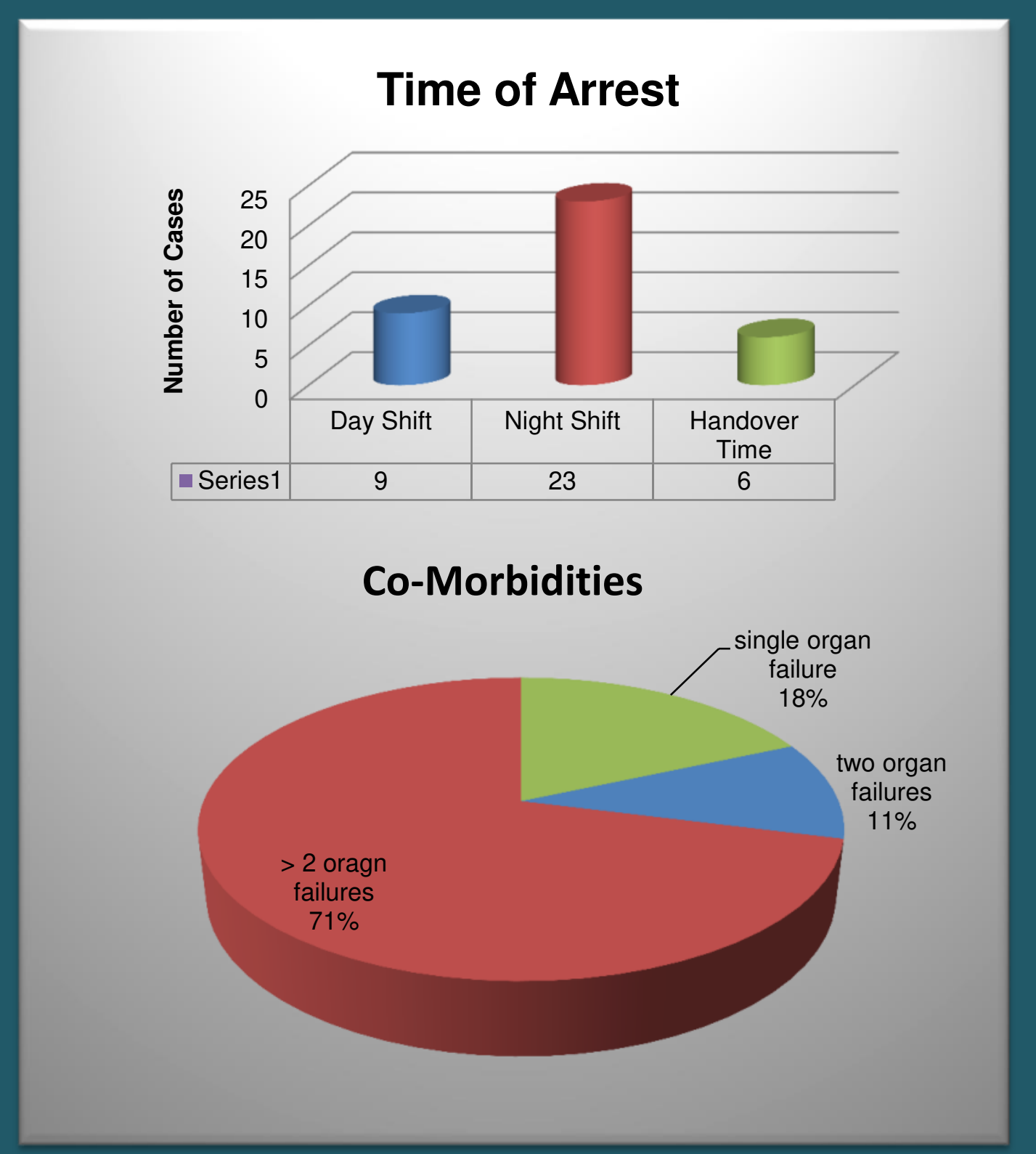

Discussion and recommendations for change

- Shortfalls in vital signs monitoring may have contributed to the number of cardiac arrests.

- In view of hospital length of stay before arrest, co-morbid status and proportion of night-time arrests it appears that mainly an appropriate treatment escalation plan had been lacking in many patients. This was also reflected in the high number of patients who after ROSC were deemed inappropriate for admission to ITU.

- The Trust has in the meantime moved to the national early warning score with a lower escalation threshold.

\section{Action Plan}

- The systematic use of in-hours treatment escalation plans should be considered to ensure appropriate patient selection.

- Application of the National Early Warning Scoring System (NEWS) in hospital wards and early referral to ITU-Outreach team accordingly.

- Re-audit incidence and potential reversible causes of in-hospital cardiac arrest.

\section{Acknowledgement:}

This project was supported by South Tyneside NHS Foundation Trust Clinical Audit Team members.

References

1) Key statistics from the National Cardiac Arrest Audit 2015/16 ICNARC. https://www.icnarc.org/DataServices/..//ef90ed89-78a2e611-80e0-1402ec3fcd79, last accessed on 16/09/2017.

2) Hogan, H (2014) the Scale and Scope of Preventable Hospital Deaths. PhD thesis, London School of Hygiene \& Tropical Medicine. DOI: 10.17037/PUBS. 01776586.

3) NCEPOD classification of care setting - Society for Acute Medicine, A SYSTEM TO MAXIMIZE LEARNING FROM MORTALITY REVIEWS ON AN ACUTE MEDICAL UNIT. Meri Alvarez. 